\title{
Full-tensor alignment criteria for sheared nematic polymers $^{\text {a) }}$
}

\author{
M. Gregory Forest ${ }^{\mathrm{b})}$ and Ruhai Zhou \\ Department of Mathematics, University of North Carolina at Chapel Hill, Chapel \\ Hill, North Carolina 27599-3250 \\ Qi Wang \\ Department of Mathematics, Florida State University, Tallahassee, Florida 32306
}

(Received 9 July 2002; final revision received 10 October 2002)

\begin{abstract}
Synopsis
The shear problem for nematic polymers consists in characterizing all stable stationary orientational distributions, steady and unsteady, versus shear rate and material parameters. Continuum theory [cf. Leslie (1968), Ericksen (1960)] provides formulas for the shear response of liquid crystals in terms of a single viscosity ratio, the Leslie tumbling parameter $\lambda_{L}$. Kuzuu and Doi $(1983,1984)$ developed a weak-flow asymptotic analysis of kinetic theory, which gives a molecular basis for all continuum theory parameters. In this paper, we develop a mesoscopic extension of the Kuzuu-Doi method, applicable to any tensor model. Our method yields orientational and rheological features of nematic polymers in weak shear with explicit formulas, parametrized by the parameters of the second-moment tensor model. This provides an explicit mesoscopic theory solution to the problem posed by Marrucci and Greco (1993) of how orientational degeneracy of quiescent nematic equilibria breaks in weak shear, leaving a finite set of steady stationary states, whose number, type (in-plane, out-of-plane), stability, phase transitions, and rheological properties scale with parameters of the model. An intriguing feature to resolve is the multiple transitions associated with distinct steady distributions (logrolling, in-plane flow alignment, out-of-plane alignment), each with its analog of the Leslie criterion $\left|\lambda_{L}\right|=1$. We illustrate our method and its physical predictions by solving the weak shear problem for the Doi quadratic closure model, whose material parameters are nematic concentration and molecular aspect ratio. The predictions are confirmed with numerical simulations of the model, and compared with experimental data in weak shear from the review article of Burghardt (1998). We further predict scaling properties due to changes in concentration and aspect ratio that are less readily available from experiments. (C) 2003 The Society of Rheology. [DOI: $10.1122 / 1.1530617]$
\end{abstract}

\section{INTRODUCTION}

The shear problem for nematic polymers is a classical topic in rheology and nonlinear anisotropic viscoelasticity, providing an experimental testbed for theory as well as a theoretical framework for experiments [cf. Kuzuu and Doi (1983, 1984), Marrucci (1991), Marrucci and Greco (1991, 1993), Larson (1998), Burghardt (1998)]. The quiescent nematic liquid is isotropic below a critical concentration, then ordered along a

\footnotetext{
a) Dedicated to Professor G. Marrucci on the occasion of his 65th birthday. The problem we address was eloquently discussed in 1993 by Marrucci and Greco, which inspired us to make an attempt at it.

b) Author to whom all correspondence should be addressed; electronic mail: forest@math.unc.edu
} 
distinguished direction: the director of Leslie-Ericksen (L-E) theory, the major director of mesoscopic tensor theory, or some measure of the peak of the probability distribution function (pdf) in kinetic theory. The standard prescription of kinetic theory is to compute the second moment of the pdf, which defines a second-order tensor and therefore a major director [Kuzuu and Doi (1983)].

Absent of an applied field, the nematic director is arbitrary, and the shear problem is to determine how this rotational degeneracy is broken. In weak shear, monodomain response is not unique; the nematic polymer liquid may be steady or unsteady, and the goal of theory is to distinguish this behavior depending on molecular or mesoscopic or continuum parameters. At the next level of detail, steady states may align in-plane or out-of-plane, and unsteady states may tumble in-plane or out-of-plane [Larson (1990), Larson and Ottinger (1991), Faraoni et al. (1999), Forest and Wang (2002), Forest et al. (2002b)]. Continuum theory [Marrucci and Grizzuti (1987)] provides a single and practical criterion in terms of the Leslie tumbling parameter, $\lambda_{L}=\left(1+\alpha_{3} / \alpha_{2}\right) /(1$ $\left.-\alpha_{3} / \alpha_{2}\right)$, depending only upon the ratio of two viscosity parameters $\alpha_{2}, \alpha_{3}$ of the L-E theory. If $\left|\lambda_{L}\right|>1$, flow-aligned steady states persist with Leslie alignment angle $\phi$ $=\frac{1}{2} \arccos \left(1 / \lambda_{L}\right)$; otherwise, some form of tumbling emerges with an explicit period formula. Thus, continuum theory predicts the stationary shear response is solely a material property; the shear rate only affects the time of approach to attracting orientational distributions. For nematic polymers, this ability to superpose all shear data in terms of strain units breaks down, which is predicted by mesoscopic and kinetic theory.

Kuzuu and Doi $(1983,1984)$ provided the key link between molecular kinetic theory and continuum theory by an asymptotic upscaling analysis in the weak-flow limit. In simple shear, their method gives $\lambda_{L}$ in terms of molecular parameters, Eq. (1) later. The Kuzuu-Doi analysis bypasses a general second-moment tensor in their quest to recover the L-E director theory and molecular descriptions of the Leslie viscosities. This kineticcontinuum link is fundamental since many experiments have been performed to rheologically characterize nematics from the L-E theory. The analysis also clarifies that the L-E theory is a leading-order balance in the uniaxial, weak-flow limit, so that one must relax those two conditions to gain further resolution of the orientational distribution. With the Smoluchowski equation of the kinetic theory, this is exceedingly difficult, as will be explained later. Nonetheless, kinetic and continuum forms of $\lambda_{L}$ are the theoretical framework from which nematic polymers are routinely classified as flow-aligning or tumbling [cf. de Gennes and Prost (1993), Mather et al. (1996a, 1996b), Burghardt (1998), Larson (1998), Ugaz et al. (2001)]. At the intermediate mesoscopic theory level, Cocchini et al. (1990) derived formulas analogous to the L-E theory for a twodimensional, planar tensor model, and several authors [Wang (1997), Rienacker and Hess (1999), Maffettone et al. (2000), Forest and Wang (2002), Grecov and Rey (2002)] have recently shown how these formulas can be derived from a planar restriction of various full-tensor models in the weak shear limit. These results give a partial solution to the weak shear problem of mesoscopic theory, giving the Leslie alignment angle of in-plane nematic states and a Leslie tumbling parameter which predicts steady-unsteady transitions. Our motivation for this paper is to have a method that definitively gives all steady states that survive orientational degeneracy in weak shear, based on a selection mechanism derived from the weak shear perturbation of the quiescent nematic state. We do not want to rely upon the correct guess (e.g., in-plane flow alignment), but rather let analytical selection criteria determine all possible director orientations that survive as stationary in the limit of weak shear flow. If this can be done analytically, then scaling properties of the selection criteria due to model parameters will follow. This was clearly the conceptual goal of Kuzuu and Doi $(1983,1984)$. 
The Kuzuu-Doi analysis is the first order in the shear rate, and posits a uniaxial second-moment tensor, focusing on how the nematic director perturbs. By design, their method does not aim to capture further relevant aspects of the shear-induced orientational distribution functions: the number of stationary distributions versus concentration; the spreading and biaxiality of the distribution, i.e., order parameters of the second-moment tensor; the stability of each steady state; and transition phenomena (in-plane to out-ofplane and steady unsteady) versus molecular parameters. In this paper we develop a mesoscopic analog of the Kuzuu-Doi asymptotic method, applicable to any secondmoment tensor model, that gains these orientational features of all steady states that survive in weak shear flow, and their explicit dependence on mesoscopic model parameters. We then deduce rheological features by evaluation of the mesoscopic stress tensor along each stable steady distribution.

We close the introduction with further comparison and contrast between our method and that of Kuzuu and Doi $(1983,1984)$. Their perturbation method is developed directly on the Doi kinetic equation, focusing on how the uniaxial director of the quiescent nematic is perturbed by weak flows. The leading-order kinetic solvability condition is a linearized, variable-coefficient, partial differential equation (pde), i.e., infinite dimensional. The variable coefficient is the linearized mean-field potential evaluated on the quiescent nematic distribution function (" $f_{0}$ "). This pde is not exactly solvable; indeed, even the variable coefficient is only known implicitly, and can only be computed numerically. Undaunted, Kuzuu and Doi $(1983,1984)$ used separation-of-variables to extract a perturbed equation for the uniaxial director of the quiescent nematic. They thereby accomplished two fundamental results:

- an implicit molecular basis for the Leslie tumbling parameter,

$$
\lambda_{L}=\frac{2 S_{2}}{\left\langle g \frac{d U_{0}}{d \theta}\right\rangle},
$$

where $S_{2}$ is the average of the second order Legendre polynomial, $g$ is the solution of a variable-coefficient ordinary differential equation [see Kuzuu and Doi (1983)], $U_{0}$ is the excluded-volume, mean-field potential evaluated on the rotationally degenerate, equilibrium distribution function; and

- molecular-based formulas for all continuum parameters (Leslie viscosities).

To get any explicit information about the full distribution, $f=f_{0}+f_{1}+\cdots$, i.e., all $f_{0}$ that survive in weak shear for all values of nematic concentration and molecular aspect ratio, and their shear-dependent correction " $f_{1}$," one must resort to numerical simulations of the full Smoluchowski equation [cf. Larson (1990), Larson and Ottinger (1991), Faraoni et al. (1999), Forest et al. (2002d)].

The method developed in this paper relies upon three features of the finite-dimensional mesoscopic approximation of kinetic theory. It is well-known how to solve the quiescent leading order problem, and explicitly parametrize the rotational symmetry of the nematic phases. Our first step is to use the weak-shear solvability conditions to determine any and all directions, in-plane or not, which survive as steady states. Second, our full-tensor solvability conditions are algebraic linear systems, and can be easily extended to any order to gain detailed information about the shear-selected stationary distributions, including the stability of each persistent state. Finally, the solvability conditions are indeed solvable, which we illustrate with the Doi closure model.

We thereby provide explicit formulas that convey orientational and rheological prop- 
erties of all stable flow-aligned states, and their phase transitions (in-plane to out-ofplane, and steady-unsteady). The formulas are explicit, so that all properties are given in terms of the Doi theory parameters, nematic concentration $N$ and molecular aspect ratio $r$. This achieves a mesoscopic version of the results that motivated Kuzuu and Doi. We also show a remarkable analytical coincidence among the transition criteria associated with the various steady shear-induced equilibria (logrolling, in-plane, and out-of-plane). These predictions and scaling properties are complementary to standard experimental data, which are almost exclusively reported for a fixed nematic liquid \{e.g., PBLG [Moldenaers and Mewis (1993)] or 5CB [Mather et al. (1996a)]\} over a range of shear rates. It is significantly more challenging to vary the molecular aspect ratio \{e.g., comparing $5 \mathrm{CB}$ and 8CB [Mather et al. (1996b)]\}, and more time consuming to vary nematic concentration (or temperature). The weak-shear analysis given here yields precisely these scaling properties of the orientation distribution and rheological features.

\section{THE DOI SECOND-MOMENT MODEL}

Let $f(\mathbf{m}, t)$ be the orientational pdf function for axisymmetric ellipsoidal molecules with axis of symmetry $\mathbf{m}$ and aspect ratio $r$. The Smoluchowski equation for $f(\mathbf{m}, t)$ is [Doi (1981), Kuzuu and Doi $(1983,1984)]$ :

$$
\begin{gathered}
\frac{D f}{D t}=\mathcal{R} \cdot\left[D_{r}(\mathbf{m})\left(\mathcal{R} f+\frac{1}{k T} f \mathcal{R} V\right)\right]-\mathcal{R} \cdot[\mathbf{m} \times \dot{\mathbf{m}} f], \\
\dot{\mathbf{m}}=\mathbf{\Omega} \cdot \mathbf{m}+a[\mathbf{D} \cdot \mathbf{m}-\mathbf{D}: \mathbf{m m m}],
\end{gathered}
$$

where $D_{r}(\mathbf{m})$ is the rotational diffusivity, $k$ is the Boltzmann constant, $T$ is the absolute temperature, $a$ is the molecular geometry parameter which plays an important role in all mesoscopic approximations of Doi kinetic theory

$$
a=\frac{r^{2}-1}{r^{2}+1} \text {. }
$$

$\mathcal{R}=\mathbf{m} \times \partial / \partial \mathbf{m}$ is the rotational gradient operator, and $D / D t(\bullet)$ denotes the material derivative $\partial / \partial t(\cdot)+\mathbf{v} \cdot \nabla(\cdot)$. In Eq. (2), the second moment of $\mathbf{m}$ with respect to the pdf;

$$
\mathbf{M}=\langle\mathbf{m m}\rangle=\int_{\|\mathbf{m}\|=1} \mathbf{m m} f(\mathbf{m}, t) d \mathbf{m},
$$

enters through the Maier-Saupe excluded-volume potential $V$ :

$$
V=-\frac{3}{2} N k T \mathbf{m m}: \mathbf{M}
$$

The mesoscopic orientation tensor $\mathbf{Q}$ is the traceless part of $\mathbf{M}$ :

$$
\mathbf{Q}=\mathbf{M}-\frac{1}{3} \mathbf{I},
$$

the fundamental mesoscopic variable which links to laboratory measurements [Burghardt (1998), Fuller (1995)]. The nematodynamical equation for $\mathbf{Q}$ is derived from Eq. (2):

$$
\begin{aligned}
\frac{d}{d t} \mathbf{Q}= & \boldsymbol{\Omega} \cdot \mathbf{Q}-\mathbf{Q} \cdot \mathbf{\Omega}+a[\mathbf{D} \cdot \mathbf{Q}+\mathbf{Q} \cdot \mathbf{D}]+\frac{2 a}{3} \mathbf{D}-2 a \mathbf{D}:\langle\mathbf{m m m m}\rangle \\
& -6 D_{r}^{0}\left[\mathbf{Q}-N\left(\mathbf{Q}+\frac{1}{3} \mathbf{I}\right) \cdot \mathbf{Q}+N \mathbf{Q}:\langle\mathbf{m m m m}\rangle\right]
\end{aligned}
$$


where $D_{r}^{0}$ is an averaged rotary diffusivity [Doi and Edwards (1986)], and the fourth moment tensor $\langle\mathbf{m m m m}\rangle$ enters due to nonlinearities. A huge literature on closure approximations exists [cf. Chaubal et al. (1995)], leading to mesoscopic models that may be related to the posited mesoscopic theories of Landau-deGennes [de Gennes and Prost (1993)] and Beris-Edwards-Grmela-Ottinger [Beris and Edwards (1994)]. The methods we introduce later can be applied to any mesoscopic $\mathbf{Q}$ tensor model. To make the analysis explicit and to compare with results from alternative methods [Forest and Wang (2002)], we choose the Doi closure which asserts

$$
(\bullet):\langle\mathbf{m m m m}\rangle \approx(\bullet): \mathbf{M M}
$$

for any second-order tensor $(\bullet)$. We also take $D_{r}^{0}$ to be constant to simplify the analysis.

We assume simple shear flow, given in Cartesian coordinates $(x, y, z)$ by

$$
\mathbf{v}=\dot{\gamma}(y, 0,0) \text {, }
$$

where $\dot{\gamma}$ is the shear rate. The rate-of-strain and vorticity tensors for Eq. (9) are

$$
\begin{gathered}
\mathbf{D}=\dot{\gamma} \tilde{\mathbf{D}}, \quad \boldsymbol{\Omega}=\dot{\gamma} \tilde{\boldsymbol{\Omega}}, \\
\tilde{\mathbf{D}}=\frac{1}{2}\left(\begin{array}{lll}
0 & 1 & 0 \\
1 & 0 & 0 \\
0 & 0 & 0
\end{array}\right), \quad \tilde{\boldsymbol{\Omega}}=\frac{1}{2}\left(\begin{array}{ccc}
0 & 1 & 0 \\
-1 & 0 & 0 \\
0 & 0 & 0
\end{array}\right) .
\end{gathered}
$$

The Peclet number Pe,

$$
\mathrm{Pe}=\frac{\dot{\gamma}}{6 D_{r}^{0}}
$$

is the shear rate normalized relative to nematic relaxation rate, and serves as the basic asymptotic parameter for weak shear.

Finally, recall that $\mathbf{M}$ and $\mathbf{Q}$ share an orthonormal frame of eigenvectors $\mathbf{n}_{i}$, called directors, with corresponding eigenvalues $d_{i}, i=1,2,3$ of $\mathbf{M}, d_{i}-\frac{1}{3}$ of $\mathbf{Q}$, called the order parameters. The director $\mathbf{n}_{i}$ associated with the maximum $d_{i}$ is the major director. Flow-aligned states are classified according to the direction of the major director relative to the shearing [flow $(x)$-flow gradient $(y)$ ] plane: in-plane if $\mathbf{n}_{i}$ lies in the $(x, y)$ plane, and then the polar angle $\phi_{L}$ is called the Leslie alignment angle; logrolling if $\mathbf{n}_{i}$ is parallel to the vorticity $(z)$ axis; and out-of-plane if $\mathbf{n}_{i}$ lies strictly between the shearing plane and vorticity axis.

\section{WEAK-SHEAR SOLVABILITY CONDITIONS}

All equilibria for the Doi closure model without flow admit the uniaxial representation

$$
\mathbf{Q}_{0}=s_{ \pm}\left(\mathbf{u} \mathbf{u}-\frac{1}{3} \mathbf{I}\right),
$$

where $s=0$ or $\mathbf{Q}=\mathbf{0}$ is the isotropic state that exists for all concentrations, and nematic states exist for sufficiently high $N \geqslant \frac{8}{3}$ with

$$
s_{ \pm}=\frac{1}{4}\left(1 \pm 3 \sqrt{1-\frac{8}{3 N}}\right),
$$


$\mathbf{u}$ is the uniaxial director which is arbitrary in the absence of an applied field; $s_{+}$indicates the upper (stable) nematic branch, and $s_{-}$labels the lower (unstable) branch.

We uniquely parametrize orientational degeneracy of the nematic phases (13), (14), by a spherical coordinate representation of $\mathbf{u} \in S_{+}^{2}$, the half-sphere consisting of the equator (shear plane) union the upper hemisphere

$$
\mathbf{u}=(\sin \theta \cos \phi, \quad \sin \theta \sin \phi, \quad \cos \theta), \quad \theta \in\left[0, \frac{\pi}{2}\right), \quad \phi \in[0,2 \pi] .
$$

When $\mathbf{u}$ lies in the shear plane $\theta=\pi / 2$, uniqueness restricts $\phi$ to any arc of $\pi$ radians, which we select as $[-\pi / 2, \pi / 2]$ following tradition.

In weak shear flows, the goal is to identify those $\mathbf{u}$ which survive as steady states versus $N$ and $a$, and to identify how this major director perturbs [Kuzuu and Doi (1983, 1984)]. For states which persist from the nematic equilibria (13), (14), then the shearinduced steady state $\mathbf{Q}$ will be given by $\mathbf{Q}_{0}$ at leading order, with an $O(\mathrm{Pe})$ correction to be determined below. To preview our method: first we will determine which $\mathbf{u} \in S_{+}^{2}$ survive as steady states; second we will exactly construct the $O(\mathrm{Pe})$ correction to each shear-selected steady state as an explicit function of the model parameters $N$ and $a$; and third, we will determine stability of each state. Finally, all alignment and rheological properties of stable steady states follow by straightforward evaluation.

In-plane and logrolling shear-alignment are each characterized either by the two constraints $Q_{x z}=Q_{y z}=0$, or by assigning one eigenvector of $\mathbf{Q}$ parallel to the vorticity axis. One must then defer to the major director to distinguish them. In our parametrization, $\theta=0$ corresponds to major director parallel to the vorticity axis; $\theta=\pi / 2(\bmod \pi)$ yields major director $\mathbf{u}$ in the shear plane; and all other choices of $\theta$ yield major director that is out-of-plane. Once special $\mathbf{Q}_{0}$ have been determined to persist in weak shear, then their major director gives a classification of in-plane, logrolling, or out-of-plane if we can show that the shear-induced correction, $\mathrm{Pe} \mathbf{Q}_{1}$, maintains that major director alignment. This is part of the method given here.

In general, the shear perturbation is expected to break the director degeneracy and the uniaxial order-parameter degeneracy of quiescent equilibria (13), (14); our goal is to determine both selection criteria. We now present the method. Recall the general biaxial representation [see Forest and Wang (2002)]:

$$
\mathbf{Q}=\sum_{i=1,2,3}\left(d_{i}-\frac{1}{3}\right) \mathbf{n}_{i} \mathbf{n}_{i}=s\left(\mathbf{n}_{1} \mathbf{n}_{1}-\frac{1}{3} \mathbf{I}\right)+\beta\left(\mathbf{n}_{2} \mathbf{n}_{2}-\frac{1}{3} \mathbf{I}\right)
$$

with $s=d_{1}-d_{3}, \beta=d_{2}-d_{3}$. Uniaxial limits are $\beta=0, s=0$, or $s=\beta$, where the uniaxial director is $\mathbf{n}_{1}, \mathbf{n}_{2}$, or $\mathbf{n}_{3}$, respectively. Biaxial states satisfy $d_{i} \neq d_{j}, \forall i, j$, with maximum $d_{i}$ defining the major director.

Assume weak shear, $\mathrm{Pe} \ll 1$, and expand $\mathbf{Q}$ in powers of Pe:

$$
\mathbf{Q}=\mathbf{Q}_{0}+\operatorname{Pe} \mathbf{Q}_{1}+\mathrm{Pe}^{2} \mathbf{Q}_{2}+\cdots
$$

At order Pe, for any mesoscopic tensor model, one gets a linearized tensor-operator equation for $\mathbf{Q}_{1}$ :

$$
\mathbf{A}\left(\mathbf{Q}_{0}\right) \cdot \mathbf{Q}_{1}=\mathbf{r}\left(\mathbf{Q}_{0}\right),
$$

where $\mathbf{A}\left(\mathbf{Q}_{0}\right)$ is the linearized quiescent nematic tensor field, and $\mathbf{r}\left(\mathbf{Q}_{0}\right)$ is the linearized flow perturbation. For the Doi closure model, these operators are given by (with $\mathbf{M}_{0}$ $\left.=\mathbf{Q}_{0}+\frac{1}{3} \mathbf{I}\right)$ : 


$$
\begin{gathered}
\mathbf{A}\left(\mathbf{Q}_{0}\right) \cdot \mathbf{Q}_{1}=\mathbf{Q}_{1}-N\left(\mathbf{M}_{0} \cdot \mathbf{Q}_{1}+\mathbf{Q}_{1} \cdot \mathbf{Q}_{0}\right)+N\left(\mathbf{Q}_{0}: \mathbf{M}_{0} \mathbf{Q}_{1}+\mathbf{Q}_{1}: \mathbf{M}_{0}+\mathbf{Q}_{0}: \mathbf{Q}_{1}\right) \mathbf{M}_{0} \\
\mathbf{r}\left(\mathbf{Q}_{0}\right)=\tilde{\mathbf{\Omega}} \cdot \mathbf{Q}_{0}-\mathbf{Q}_{0} \cdot \tilde{\mathbf{\Omega}}+a\left(\tilde{\mathbf{D}} \cdot \mathbf{Q}_{0}+\mathbf{Q}_{0} \cdot \tilde{\mathbf{D}}\right)+a\left(\frac{2}{3} \tilde{\mathbf{D}}-2 \tilde{\mathbf{D}}: \mathbf{M}_{0} \mathbf{M}_{0}\right)
\end{gathered}
$$

In the analysis deferred to Appendix B, we utilize the Cartesian components of $\mathbf{Q}_{1}$ to convert the tensor-operator Eq. (18) to a transparent linear system

$$
A \cdot Q_{1}=r
$$

where $A$ is a $5 \times 5$ matrix, $Q_{1}=\left[Q_{1}(x x), Q_{1}(x y), Q_{1}(x z), Q_{1}(y y), Q_{1}(y z)\right]^{T}$ comprises the five components of $\mathbf{Q}_{1}$, and $r$ is a five-vector derived from $\mathbf{r}$, Eq. (20).

As in Kuzuu and Doi (1983), the Fredholm alternative theorem provides the weakshear solvability condition. Those $\mathbf{Q}_{0}$, defined in Eqs. (13), (14), uniquely parametrized by $\mathbf{u} \in S_{+}^{2}$ from (15), which remain steady through $O(\mathrm{Pe})$ are characterized by

$$
r\left(\mathbf{Q}_{0}\right) \perp \mathcal{N}\left[A^{T}\left(\mathbf{Q}_{0}\right)\right],
$$

where $\mathcal{N}$ denotes the null space of the matrix $A^{T}$. This solvability condition determines which quiescent states (13) (14) persist in weak shear, including those arising from the stable and unstable nematic equilibrium branches (14), and the isotropic state.

To completely construct $\mathbf{Q}_{1}$ for each persistent $\mathbf{Q}_{0}$, to determine stability and out-ofplane transitions versus mesoscopic parameters, we require another order of perturbation. That is, we need the solvability condition for $\mathbf{Q}_{2}$. The tensor equation for $\mathbf{Q}_{2}$ and corresponding orthogonality condition are given in Appendix A.

\section{STEADY STATES IN WEAK SHEAR}

We report the physical conclusions from an exact analysis of the solvability conditions for $\mathbf{Q}_{1}$ and $\mathbf{Q}_{2}$, Eqs. (22) and (A3). We give the construction of $\mathbf{Q}$ through order $O(\mathrm{Pe})$ for all persistent steady states, first those associated with the quiescent isotropic state, followed by the two nematic branches. We then deduce directors and order parameters from the tensor representation (16), and finally display the alignment and rheological properties of all stable steady states. The mathematical analysis is summarized in Appendix B.

\section{A. The shear-perturbed, nearly isotropic branch}

The isotropic steady state perturbs at $O(\mathrm{Pe})$ to a remarkably simple form

$$
\mathbf{Q}=\mathbf{0}+\mathrm{Pe} \frac{2 a}{3-N} \tilde{\mathbf{D}}+O\left(\mathrm{Pe}^{2}\right)
$$

where $\tilde{\mathbf{D}}$ is the normalized rate-of-strain (11) for pure shear. The directors of $\mathbf{Q}$ are explicit

$$
\mathbf{n}_{1}=(1,-1,0), \quad \mathbf{n}_{2}=(1,1,0) \quad \mathbf{n}_{3}=(0,0,1),
$$

with corresponding distinct order parameters

$$
d_{1}=\frac{1}{3}+\frac{a}{N-3} \mathrm{Pe}, \quad d_{2}=\frac{1}{3}-\frac{a}{N-3} \mathrm{Pe}, \quad d_{3}=\frac{1}{3} .
$$

All features of the shear-perturbed isotropic state follow:

- a unique steady state survives for $N \neq 3$; 
- it is biaxial $\left(d_{i} \neq d_{j}\right.$, if $\left.i \neq j\right)$;

- the major director is always in-plane;

- for rods $(a>0)$, the Leslie alignment angle $\phi$, to within $O\left(\mathrm{Pe}^{2}\right)$, is universal, independent of aspect ratio parameter $a$ and shear rate Pe:

$-45^{\circ}$ above the flow direction when $N<3$, and

$-45^{\circ}$ below the flow direction when $N>3$;

- the sign of $\phi$, i.e., direction of tilt relative to the flow direction, reverses for discotics $(a<0)$.

- at higher order in Pe, the director tilts toward the flow axis for rods and discotics which follows from an explicit $O\left(\mathrm{Pe}^{2}\right)$ construction of $\mathbf{Q}_{2}$ :

$$
\mathbf{Q}_{2}=-\frac{3 a}{(3-N)^{3}}\left(\begin{array}{ccc}
a+3-N & 0 & 0 \\
0 & a-3+N & 0 \\
0 & 0 & -2 a
\end{array}\right) .
$$

From the next order solvability condition, stability is straightforward with explicit linearized eigenvalues

$$
\lambda_{1} \approx \lambda_{2} \approx \lambda_{3} \approx \lambda_{4} \approx \lambda_{5}=-\left(1-\frac{N}{3}\right)+O(\mathrm{Pe})
$$

Therefore, the shear-perturbed isotropic branch is stable for $N<3$, and unstable for $N>3$, as expected. We depict the region of stable nearly isotropic states in $(a, N)$ parameter space in Fig. 1(b), which summarizes all stable flow aligned states. In Fig. 5, the $O(\mathrm{Pe})^{2}$ construction is used to graph the stable alignment angle $\phi$ for dilute concentrations, together with other stable alignment states.

We note that this asymptotic construction breaks down for $|N-3|=O(\mathrm{Pe})$, i.e., the solvability Eq. (22) does not have a solution. This implies an $O(\mathrm{Pe})$ gap in concentrations $N \approx 3$ where the isotropic branch fails to persist; this result is confirmed by mesoscopic numerical simulations [Wang (1997), Forest et al. (2002a)], and is consistent with kinetic theory [Forest et al. (2002c)].

\section{B. The stable nematic equilibrium branch}

Consider the upper nematic solution $\mathbf{Q}_{0}$ with order parameter $s_{+} \in\left[\frac{1}{4}, 1\right)$, Eqs. (13) and (14), which we abbreviate as $s$ in the formulas later. Note the order parameter $\frac{1}{4}$ $\leqslant s \leqslant 1$ is a monotone function of the concentration $\frac{8}{3} \leqslant N \leqslant \infty$, and we refer to $s$ and $N$ interchangeably in discussions to follow. Recall $\mathbf{u} \in S_{+}^{2}$, Eq. (15), gives a 1:1 parametrization of rotational symmetry, which we decompose into three disjoint cases defined by the polar angle $\theta$ : vorticity alignment, shear-plane alignment, and out-of-plane alignment. For each family of persistent equilibria in weak shear, we also assess their linearized stability. Recall that quiescent nematic states always have 2 zero linearized eigenvalues corresponding to orientational degeneracy, so that the stability analysis amounts to determining how those zero eigenvalues perturb for each persistent steady state. 

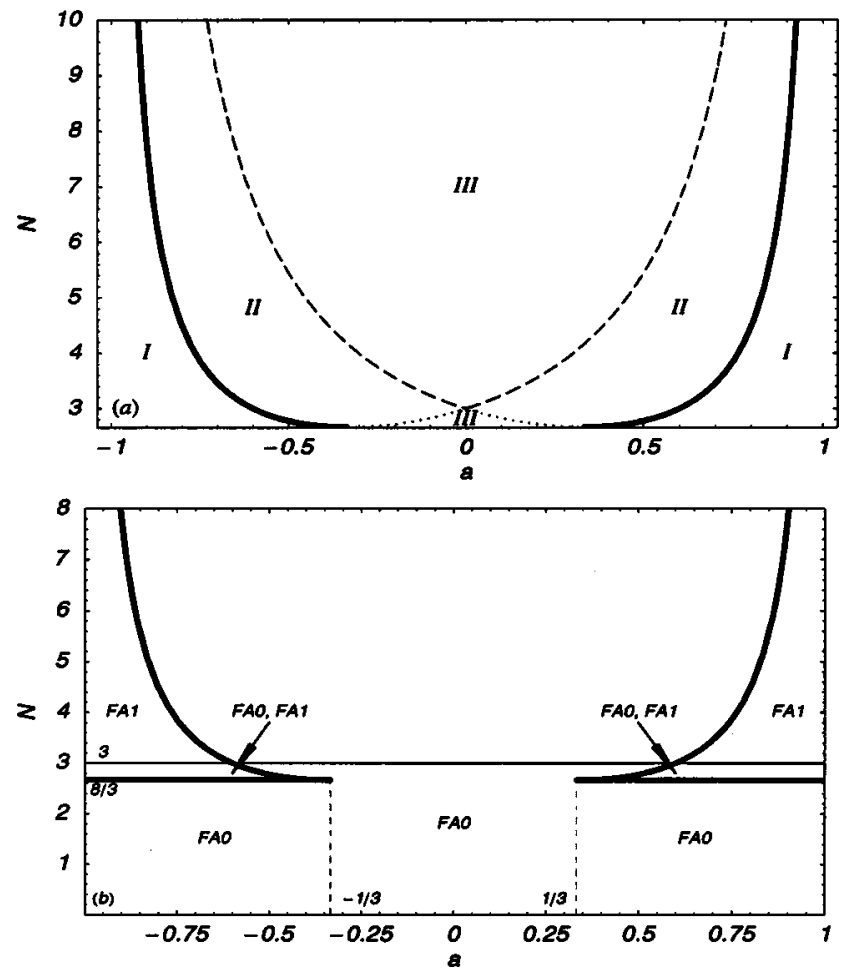

FIG. 1. (a) Regions in $(a, N)$ space of shear-aligned nematic steady states. See Table I for specific types of steady states for each region. The solid boundary between region (I) and (II) corresponds both to loss of the stable flow-aligning state and existence of the out-of-plane state, where the mesoscopic Leslie tumbling parameter $\lambda_{L}=[a(2+s)] / 3 s$ has modulus 1. (b) Regions of all stable steady states, both nearly isotropic (FA0) and nematic (FA1). (Stable logrolling states only emerge for $N \gg 1$.)

\section{Shear perturbed logrolling states: $\theta=0$}

The perturbed solution $\mathbf{Q}$ corresponding to $\mathbf{u}=(0,0,1)$ is explicitly constructed

$$
\mathbf{Q}=\frac{s}{3}\left(\begin{array}{ccc}
1 & 0 & 0 \\
0 & 1 & 0 \\
0 & 0 & -2
\end{array}\right)+\operatorname{Pe} \frac{a(1-s)^{2}(1+2 s)}{9 s}\left(\begin{array}{ccc}
0 & 1 & 0 \\
1 & 0 & 0 \\
0 & 0 & 0
\end{array}\right)+O\left(\mathrm{Pe}^{2}\right),
$$

TABLE I. Shear-selected steady states in weak shear for each region in Fig. 1. FA0 is the unique flow-aligning steady state selected from the quiescent isotropic state. Others are selected from quiescent nematic equilibria. FA indicates in-plane flow-alignment states, LR logrolling states, OS out-of-plane steady states. "+ " indicates states that survive from the upper nematic branch, "- " the lower nematic branch. "1", "2" label multiplicity. For example, six steady nematic states and a nearly isotropic steady state exist in region (I).

\begin{tabular}{cc}
\hline \hline I & FA0, FA $_{+}^{1,2}, \mathbf{F A}_{-}^{1,2}, \mathbf{L} \mathbf{R}_{ \pm}$ \\
II & $\mathbf{F A 0}, \mathbf{F A}_{-}^{1,2}, \mathbf{L} \mathbf{R}_{ \pm}$ \\
III & $\mathbf{F A 0}, \mathbf{L} \mathbf{R}_{ \pm}$ \\
Solid curve & $\mathbf{F A 0}_{\mathbf{O}}^{1,2}, \mathbf{L R}_{ \pm}$ \\
Dashed curve & $\mathbf{F A 0}_{+}^{1,2}, \mathbf{L S}_{ \pm}^{1,2}$ \\
Dotted curve & $\mathbf{F A 0}, \mathbf{O S}_{-}^{3,4}, \mathbf{L R}_{ \pm}$ \\
\hline \hline
\end{tabular}


with distinct order parameters at $O(\mathrm{Pe})$ :

$$
\begin{gathered}
d_{1}=\frac{2 s+1}{3}, \\
d_{2}=\frac{1-s}{3}-a \mathrm{Pe} \frac{1-3 s^{2}+2 s^{3}}{3 s}, \\
d_{3}=\frac{1-s}{3}+a \mathrm{Pe} \frac{1-3 s^{2}+2 s^{3}}{3 s},
\end{gathered}
$$

and corresponding directors

$$
\mathbf{n}_{1}=(0,0,1), \quad \mathbf{n}_{2}=(1,-1,0), \quad \mathbf{n}_{3}=(1,1,0),
$$

respectively. All features of logrolling equilibria in weak shear follow:

- logrolling states always exist, for all nematic concentrations $N>\frac{8}{3}$ and aspect ratios $r$

- the major directors of $\mathbf{Q}_{0}$ and $\mathbf{Q}_{1}$ align with the vorticity axis, i.e., the shear-selected state is logrolling through $O(\mathrm{Pe})$;

- the equilibrium is biaxial, $d_{i} \neq d_{j}$ for $i \neq j$, with maximum degree of order $d_{1}$ $=(2 s+1) / 3$ that remains constant through $O(\mathrm{Pe})$;

- the minor directors are universal, similar to the isotropic state, in the sense that they are aligned at $45^{\circ}$ in the shear plane independent of concentration $N$ and shape parameter $a$. These properties follow because $\mathbf{Q}_{1}$ is proportional to the rate-of-strain tensor $\tilde{\mathbf{D}}$, from Eq. (28).

This recovers the result from Forest and Wang (2002), by a completely different method, that the Doi closure preserves vorticity-aligning steady states for all aspect ratios and nematic concentrations. The present method also shows the Hinch-Leal closures [Hinch and Leal (1976)] also yield unconditional preservation of logrolling equilibria, whereas the Tsuji-Rey closure [Tsuji and Rey (1997)] has one isolated concentration, independent of $a$, where the logrolling solvability condition fails. Aside from this outlier, these results agree with numerical results of the Doi kinetic theory with infinite aspect ratio [Forest et al. (2002c)]. A referee has suggested that a symmetry argument implies logrolling states must always exist in shear, but such a result eludes us.

The next issue is stability of logrolling equilibria in weak shear. Numerical simulations [Forest and Wang (2002), Forest et al. (2002d)] reveal extreme sensitivity i.e., contradictory predictions, in the stability of logrolling states for various closure rules: Doi and Tsuji-Rey closures predict instability except at very high concentrations, while HinchLeal closures predict unconditional stability. Numerical results of kinetic theory with infinite aspect ratio [Forest et al. (2002c)] show that stability of logrolling equilibria in weak shear is concentration dependent: instability for all intermediate nematic concentrations, then stability above a critical concentration. We now use our method to assess logrolling stability for the Doi closure, which reveals a delicate dependence on concentration. The result is:

All logrolling states are unstable in weak shear for the Doi closure model, except possibly in the extreme concentration limit $N \gg 1$. Stability is determined by the two linearized eigenvalues which break orientational degeneracy, i.e., that are zero absent of shear. To get this level of detail, we have to analyze the solvability conditions through 
second order in the asymptotic expansion, which is beyond any previous kinetic or mesoscopic analysis. Omitting all details, we deduce the pair of out-of-plane linearized eigenvalues for any logrolling state

$$
\pm \frac{\mathrm{Pe}}{2} \sqrt{\left[\frac{a(2+s)}{3 s}\right]^{2}-1}+\frac{\mathrm{Pe}^{2}}{27}\left[\frac{a}{s}(1+2 s)(1-s)\right]^{2} .
$$

We summarize consequences of these exact stability formulas:

- First note that the $O\left(\mathrm{Pe}^{2}\right)$ term is positive definite for finite concentrations, $N<\infty$, and nonspherical molecules $a \neq 0$. This implies at least a one-dimensional instability of all logrolling states, as follows.

- If $|a(2+s) / 3 s|<1$, or equivalently from Eq. (14), for $N$ sufficiently large, $N$ $>(3-|a|)^{2} / 3\left(1-a^{2}\right)$, the $O(\mathrm{Pe})$ terms are purely imaginary, and stability defers to the $O\left(\mathrm{Pe}^{2}\right)$ terms, which we have just noted are positive definite. For all $N, a$ satisfying this inequality, logrolling equilibria are weakly unstable (with dimension 2).

- If $|a(2+s) / 3 s|>1$, or $\frac{8}{3}<N<(3-|a|)^{2} / 3\left(1-a^{2}\right)$, an $O(\mathrm{Pe})$, one-dimensional instability exists for all logrolling equilibria.

- The transition boundary, $|a|(2+s) / 3 s=1$ or $N=(3-|a|)^{2} / 3\left(1-a^{2}\right)$, has 2 zero eigenvalues at leading order, which is the harbinger of a bifurcation of some kind in the full-tensor equation. However, it is not a stability transition of the logrolling states, only a jump in the dimension and strength of the instability.

- This transition criterion, $\operatorname{sgn}[|a(2+s) / 3 s|-1]$, or $\operatorname{sgn}\left[N-(3-|a|)^{2} / 3\left(1-a^{2}\right)\right]$, will be derived for two other classes of equilibria to follow: the steady-unsteady transition of in-plane flow alignment, and the existence of out-of-plane steady states. Said differently, the quantity $a(2+s) / 3 s$ shall emerge as the mesoscopic transition parameter for all persistent nematic equilibria!

- In the extreme concentration limit, $N \gg 1$ or $s \approx 1$, these eigenvalue estimates vanish. If we let $1-s=O(\mathrm{Pe})$, then the $O(\mathrm{Pe})$ terms are purely imaginary, and the next order term becomes $O\left(\mathrm{Pe}^{4}\right)$. Therefore, the cubic terms become dominant, which we do not take pains to compute analytically. Rather, numerical simulations show a supercritical Hopf bifurcation for $N \gg 1$ and all $|a|<1$, at which the logrolling equilibrium goes from unstable to stable [Forest et al. (2002a)].

- To summarize the solvability analysis of logrolling states: they exist and are unstable for all finite $N$ and $a \neq 0$; a bifurcation of some kind is detected at $N=$ (3 $-|a|)^{2} / 3\left(1-a^{2}\right)$, beyond which the logrolling instability becomes an order of magnitude weaker, and another bifurcation is detected for $N$ large where the logrolling state may stabilize. These features are confirmed by numerical simulations of the Doi closure model, so our method is accurate within the precision of asymptotic analysis. Furthermore, these qualitative bifurcations occur in the Doi kinetic theory in weak shear [Forest et al. (2002c)]; the Doi closure introduces errors by pushing the stability of the logrolling state to extremely high concentrations.

\section{Shear perturbed in-plane flow-aligned states: $\theta=\pi / 2$}

An in-plane major director (15) reduces to $\mathbf{u}_{0}=(\cos \phi, \sin \phi, 0)$ for some angle $\phi$. Analysis of the solvability conditions shows that flow-alignment requires sufficiently extreme molecule aspect ratios $r$ and nematic, but not too high, concentrations $N$ :

- for $1 / \sqrt{2}<r<\sqrt{2}$, i.e., $|a|<\frac{1}{3}$, in-plane flow-alignment does not occur;

- if $\sqrt{2}<r<\infty$ or $0<r<1 / \sqrt{2}$, i.e., $\frac{1}{3}<|a|<1$, flow-aligned steady states exist for a finite range of nematic concentrations, $\frac{8}{3}<N \leqslant(3-|a|)^{2} / 3\left(1-a^{2}\right)$; 
when these conditions are met, our construction gives the Leslie alignment angle $\phi_{L}$ :

$$
\cos 2 \phi_{L}=\frac{3 s}{a(s+2)}=\frac{1+3 \sqrt{1-\frac{8}{3 N}}}{a\left(3+\sqrt{\left.1-\frac{8}{3 N}\right)}\right.},
$$

which is equivalent to formulas derived by Cocchini et al. (1990), Wang (1997), Rienacker and Hess (1999), Maffettone et al. (2000), Forest and Wang (2002), and Grecov and Rey (2002) for various tensor models with posited in-plane tensors in weak shear.

- If $r=\sqrt{2}$ or $r=1 / \sqrt{2}$, i.e., $|a|=\frac{1}{3}$, flow-alignment only exists at $N=\frac{8}{3}$, with $\phi$ $=0$ for $a=\frac{1}{3}, \phi=\pi / 2$ for $a=-\frac{1}{3}$.

In regimes where flow-aligned states exist, the perturbed solution $\mathbf{Q}$ is explicitly constructed to $O(\mathrm{Pe})$ as

$$
\mathbf{Q}=s\left(\mathbf{u}_{0} \mathbf{u}_{0}-\frac{\mathbf{I}}{3}\right)+\operatorname{Pe}\left[c_{1} \tilde{\mathbf{D}}+c_{2}\left(\begin{array}{ccc}
1 & 0 & 0 \\
0 & 1 & 0 \\
0 & 0 & -2
\end{array}\right)+c_{3}\left(\begin{array}{ccc}
1 & 0 & 0 \\
0 & -1 & 0 \\
0 & 0 & 0
\end{array}\right)\right]+O\left(\mathrm{Pe}^{2}\right),
$$

where $s$ is fixed by $N$, Eq. (14), $\mathbf{u}_{0}$ is given above, $\phi=\phi_{L}$ is given by Eq. (34), and

$$
\begin{gathered}
c_{1}=\frac{(1-s)^{2}(1+2 s)}{9 s(4 s-1)}\left[a(11 s+4)-\frac{9 s^{2}(19 s+8)}{a(2+s)^{2}}\right], \\
c_{2}=\frac{(1-s)^{2}(1+2 s) \tan 2 \phi}{6(4 s-1)}, \\
c_{3}=\frac{3 s(1-s)^{2}(1+2 s)(19 s+8) \tan 2 \phi}{6 a(2+s)^{2}(4 s-1)} .
\end{gathered}
$$

The unsteady transition of in-plane flow-aligned states follows from equation Eq. (34), exactly the condition derived above in the stability analysis of logrolling equilibria

$$
N_{*}(a)=\frac{(3-|a|)^{2}}{3\left(1-a^{2}\right)} \text {. }
$$

This boundary is the solid curve in Fig. 1(a), which separates the parameter space $(a, N)$ of the Doi closure model into regions where various flow-aligned states exist or not. Boundaries for other steady states arising from nematic equilibria are also given. Recall from the analysis above that unstable logrolling states exist across the entire region. Figure 1(a) and Table I therefore display all persistent (stable and unstable) steady states in the weak shear limit for all mesoscopic parameters $(a, N)$.

To illustrate the information contained in region (I) in Fig. 1(a), consider three distinct aspect ratios corresponding to $a=0.7,0.8,0.9$. Our theory predicts flow-aligned stable nematic states exist for $\frac{8}{3}<N<\frac{529}{153}, \frac{8}{3}<N<\frac{121}{27}, \frac{8}{3}<N<\frac{147}{19}$, respectively, whose explicit Leslie alignment angle is graphed in Fig. 2. We also show the alignment angle $\phi_{L}$ for three distinct nematic concentrations $N=3,4,10$, which reveals the scaling 
behavior of in-plane alignment versus molecular aspect ratio, and the limiting behavior as these states cease to exist. This region in Fig. 1(a), as shown, has five other steady nematic states, all unstable, as well as a nearly isotropic unstable state. (Figure 5 extends to the dilute regime where the nearly isotropic stable alignment state exists.)

We note that this critical nematic concentration $\left(N_{*}\right)$ for each extreme aspect ratio, Eq. (36), varies with closure approximation. However, the existence of a cutoff condition like Eq. (36) is robust to closure as determined from different methods in [Forest and Wang (2002)]. From Fig. 2, one concludes certain robust features:

- at the onset of quiescent nematic equilibria, $N=\frac{8}{3}$, in-plane shear-aligned states begin with Leslie angle $\phi_{L}=\frac{1}{2} \cos ^{-1}(1 / 3 a)$, which is nearly constant across a huge aspect ratio range [the starting point of each curve in Fig. 2(a) varies only by a couple degrees];

- the range of concentrations over which stable in-plane alignment persists is strongly dependent on the aspect ratio parameter $a$, with explicit scaling law Eq. (36);

- all shear-aligned states terminate at a critical concentration $N_{*}$ with zero Leslie angle, $\phi_{L}=0$;

- note the $O(\mathrm{Pe})$ eigenvalues $\lambda_{2,5}$ both vanish at $N=N_{*}$, signalling the onset of an out-of-plane instability as the flow-aligned state terminates, and a bifurcation of some kind. These conditions are consistent with two phenomena: an out-of-plane steady transition, or a steady-unsteady in-plane transition. Both occur: the former we derive below, the latter is confirmed by numerical simulations or the unsteady analysis in [Cocchini et al. (1990), Wang (1997), Rienacker and Hess (1999), Maffettone et al. (2000), Forest and Wang (2002)].

When the alignment condition (34) is satisfied, below the solid curves in Fig. 1(a), two in-plane Leslie alignment angles $\phi_{ \pm}$are selected; we now determine which is stable, and when any bifurcations may occur (from zero linearized eigenvalues). The linearized eigenvalues of Eqs. (7) and (8) for either equilibria are given by

$$
\lambda_{1}=-\frac{3 s}{(1-s)(1+2 s)}+O(\mathrm{Pe})
$$
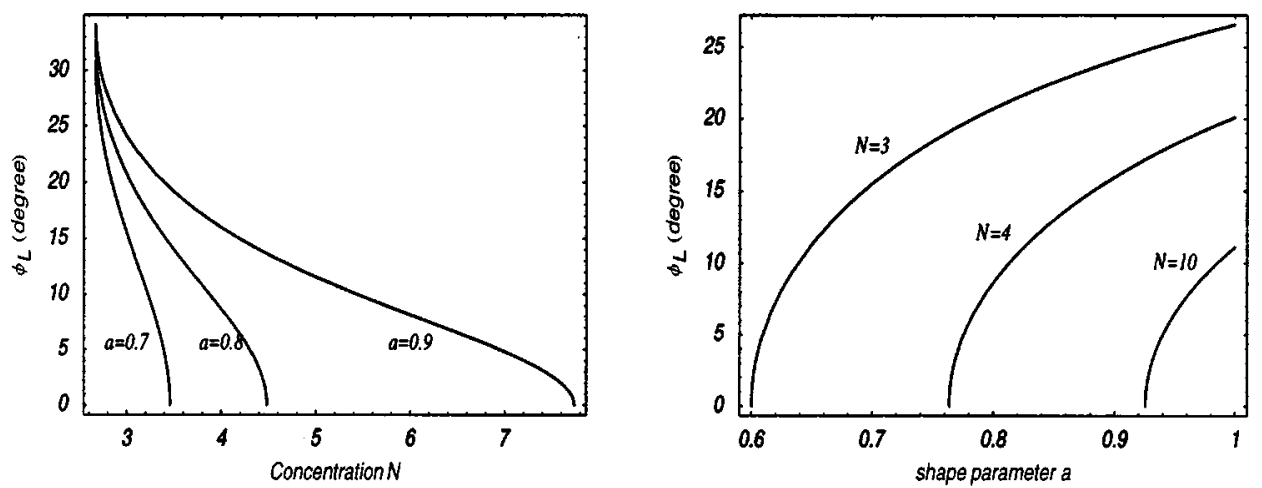

FIG. 2. Explicit alignment properties of stable in-plane states from region I, Fig. 1(a). Left: The Leslie alignment angle $\phi_{L}$ for stable in-plane steady solutions along three vertical slices of Fig. 1(a) corresponding to aspect ratio parameters $a=0.7,0.8,0.9$. Right: $\phi_{L}$ for three horizontal slices of Fig. $1(\mathrm{a}), N=3,4,10$. 


$$
\begin{gathered}
\lambda_{2}=-\mathrm{Pe} \frac{a(2+s)(1+5 s)}{6 s(4 s-1)} \sin 2 \phi+O\left(\mathrm{Pe}^{2}\right), \\
\lambda_{3}=-\frac{3 s}{(1-s)(1+2 s)}+O(\mathrm{Pe}), \\
\lambda_{4}=-\frac{s(4 s-1)}{(1-s)(1+2 s)}+O(\mathrm{Pe}), \\
\lambda_{5}=-\mathrm{Pe} \frac{a(2+s)}{3 s} \sin 2 \phi+O\left(\mathrm{Pe}^{2}\right) .
\end{gathered}
$$

$\lambda_{2}$ and $\lambda_{5}$ characterize the shear-broken zero eigenvalues of orientational degeneracy, from which stability follows if and only if $\operatorname{sgn}(a \phi)>0$. We conclude: for rods, the stable Leslie alignment angle is always positive; for discotics, the stable alignment angle is negative. The rod-discotic correspondence (rods and discotics of reciprocal aspect ratio have the same alignment properties but with a rotation by $\pi / 2$ radians in the shear plane) also follows by a symmetry, given in Forest and Wang (2002) for mesoscopic theory and in Forest et al. (2002b) for kinetic theory.

Finally, Eq. (34) gives the in-plane quiescent directors that will survive the shear perturbation. The shear-dependent correction, $\tilde{\phi}(\mathrm{Pe})$, can also be found explicitly

$$
\cos 2 \tilde{\phi}=\cos 2 \phi+\mathrm{Pe} \frac{4(1-s)^{2}(1+2 s)^{2} \sin 2 \phi}{3 s(2+s)(4 s-1)}+O\left(\mathrm{Pe}^{2}\right) .
$$

Simple calculus on this formula implies $\tilde{\phi} \leqslant \phi$ for $0<$ Pe $\ll 1$, i.e., the major director of in-plane rods and discotics always moves toward the flow axis with increased shear rate. We note that this prediction of Doi theory arises precisely in the L-E limit, yet this Pe-scaling behavior cannot be captured by the L-E theory. We now compare these predictions with data from [Burghardt (1998)], Figs. 5(a), 5(b), 7(a), 7(b) for the Pe dependence of the Leslie alignment angle and birefringence. (Later we give rheological comparisons.) We choose a fixed nematic concentration $N=7.7$ and rod-like aspect ratio $a=0.9(r \approx 4.4)$, Fig. 3, so that the Doi model flow-alignment angle is consistent with the reported data. Qualitative agreement is evident. The exact formulas also predict scaling behavior with respect to the other model parameters, $N$ and $a$, which we convey in Fig. 4. The left figure shows that at lower nematic concentrations the alignment angle is tilted significantly from the flow axis, while the right figure shows the alignment angle increases with aspect ratio for the same concentration and shear rate. In Fig. 5, we superimpose the full concentration range of stable in-plane alignment states, Fig. 1(b), including the nearly isotropic states (FA0) and shear-perturbed nematic states (FA1). Note the narrow band of bi-stability, $\frac{8}{3}<N<3-O(\mathrm{Pe})$, which persists from the quiescent isotropic-nematic transition. These results agree qualitatively with numerical results of kinetic theory [Forest et al. (2002c)].

\section{Out-of-plane flow-aligned states: $0<\theta<\pi / 2$}

Analysis of our solvability conditions shows pairs of out-of-plane solutions, mirror symmetric with respect to the shearing plane, are selected in the weak shear limit provided two conditions are satisfied: 

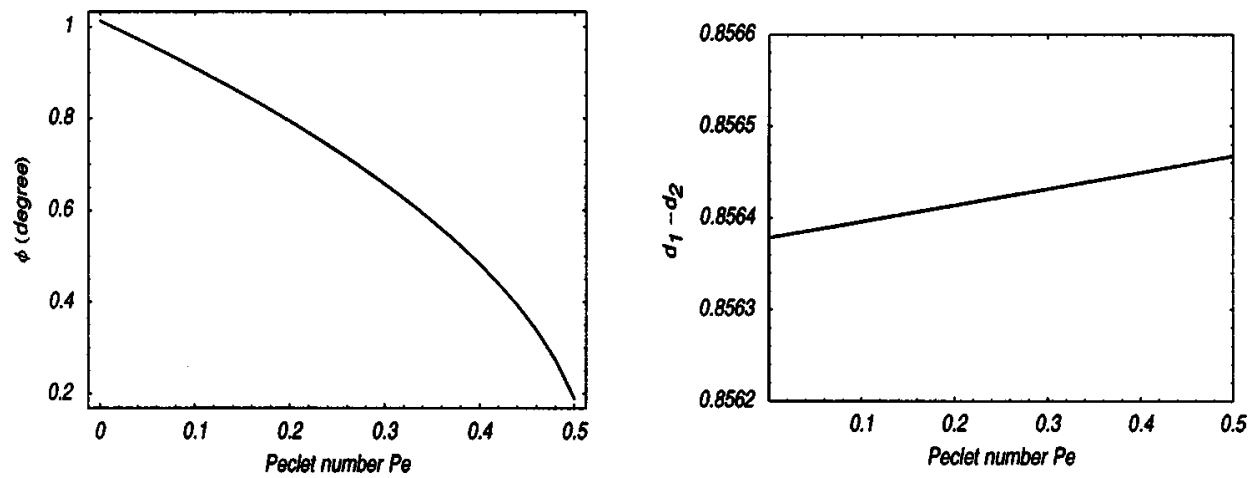

FIG. 3. Theoretical predictions of shear-rate scaling behavior for the Leslie alignment angle $\phi_{L}$ and in-plane birefringence $d_{1}-d_{2}$. Nematic concentration $N=7.7$ and aspect ratio parameter $a=.9$ are chosen so that $\phi_{L}$ is nearly parallel to the flow-axis, which allows comparison with the data in Burghardt (1998).
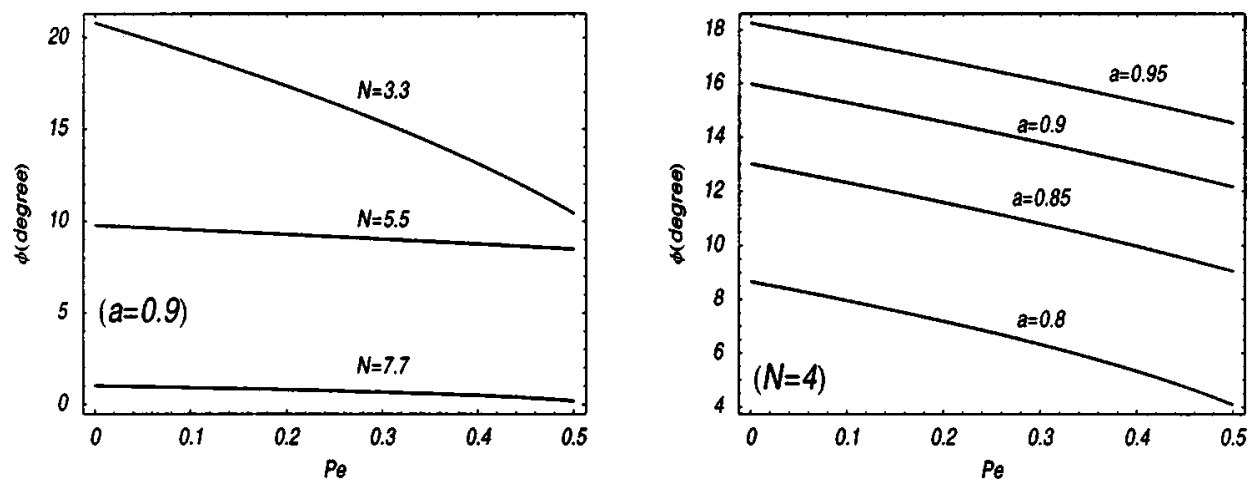

FIG. 4. Theoretical predictions of scaling behavior of the in-plane alignment angle $\phi_{L}$ for variable concentration $N$ and molecular aspect ratio parameter $a$.

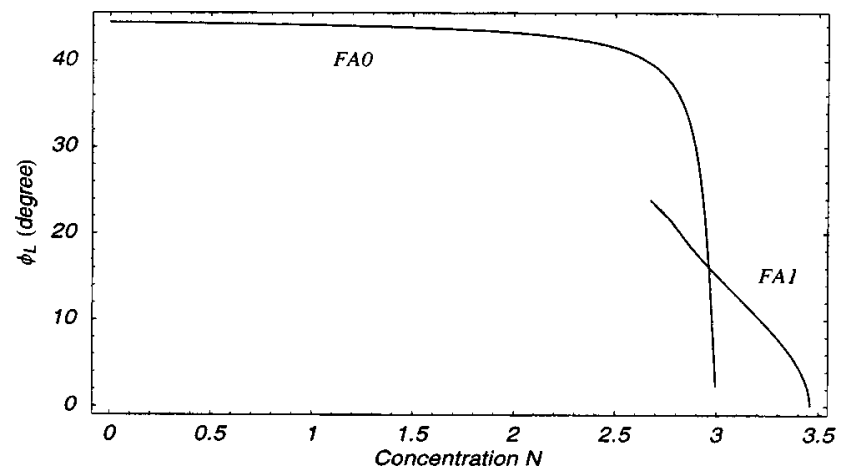

FIG. 5. The in-plane alignment angle of the nearly isotropic (FA0) and nematic (FA1) steady states from Fig. 1 (b), corresponding to the vertical slice $a=0.7$, with $\mathrm{Pe}=0.01$. The concentration $N$ varies from the dilute regime up to the solid curve that separates regions I and II, where stable steady states cease. 
- the aspect ratio must be sufficiently non-spherical, but finite: $\frac{1}{3} \leqslant|a|<1$; and

- the concentration must be a unique finite value

$$
N=\frac{(3-|a|)^{2}}{3\left(1-a^{2}\right)} .
$$

Comparison of Eqs. (36) and (43) shows out-of-plane steady states exist precisely as the in-plane flow-aligning states disappear with Leslie angle $\phi=0$; it is also precisely where the logrolling instability goes from strong $O(\mathrm{Pe})$ to weak $O\left(\mathrm{Pe}^{2}\right)$. While these states are not experimentally observable, they have a fundamental implication as to whether the steady-unsteady phase transition at weak shear rates is first or second order! The literature addresses the flow-aligning to tumbling transition, but this is not the mesoscopic [Forest and Wang (2002)] nor the kinetic [Forest et al. (2002c)] Doi model transition scenario. The tumbling solution that emerges in weak shear is unstable to out-of-plane perturbations; this is already suggested by our stability analysis for the in-plane aligned states as $N \rightarrow(3-|a|)^{2} / 3\left(1-a^{2}\right)$. The stable periodic state above this concentration in weak shear is the out-of-plane kayaking state. Thus, the pair of out-ofplane stable steady states serve an intriguing role to deliver the major director from in-plane along the flow axis, all the way to nearby the vorticity axis over an extremely small interval of concentrations; they then destabilize and vanish, giving way to the kayaking oscillation around the vorticity axis.

This analysis confirms another symmetry discussed in [Faraoni et al. (1999), Chillingworth et al. (2001), Forest et al. (2002b), Forest and Wang (2002)]: mirror-reflection about the shear plane. Notice that $(\theta, \phi)$ and $(\pi-\theta, \pi+\phi)$ correspond to the same director. Take $\frac{1}{3} \leqslant a<1$ for example: two out-of-plane solutions (for a specific $\theta$ ) survive when $\phi=0$ and $\phi=\pi$. $(\theta, 0)$ and $(\theta, \pi)$ [equivalent to $(\pi-\theta, 0)$ ] are in fact symmetric about the shear plane $(\theta=\pi / 2)$. If we vary $\theta$, we get a half circle of directors in the $x-z$ plane that connects to the logrolling state $(\theta=0)$ and the flowaligning state $(\theta=\pi / 2)$. This half circle of directors corresponds to the vertical lines shown in Figs. 9 and 10 for small Pe. As Pe becomes larger, this vertical line opens into a circle; see the earlier references.

\section{Lower nematic solution branch}

The same analysis outlined earlier can be applied to the lower quiescent nematic branch, $s_{-}$in Eq. (14). Since these equilibria are all unstable at rest, they remain unstable in weak shear. Only at higher shear rates can (and do) unstable-stable transitions occur, but that is beyond this analysis. Persistence of these states is necessary for our stated purpose to give a complete accounting of all stable and unstable stationary distributions that survive in weak shear for the Doi closure. We shall just summarize the conclusions, which are similar to the upper nematic branch, with a few exceptions. The results determine the dashed and dotted boundaries of Fig. 1, and the subscript "-" steady states labeled in Table I.

Logrolling states always persist for all $N$ and $a$. In-plane flow-aligning states from the lower nematic equilibria exist in larger parameter regions, including regions I and II in Fig. 1(a). For $|a| \geqslant \frac{1}{3}$, where upper nematic aligned states also exist, the lower nematic alignment region extends from $N=\frac{8}{3}$ all the way to the dashed curve that depicts one boundary of regions II and III:

$$
N_{-}(a)=\frac{(3+|a|)^{2}}{3\left(1-a^{2}\right)} \text {. }
$$


For more spherical aspect ratios, $|a|<\frac{1}{3}$, upper nematic alignment states do not exist but lower nematic alignment occurs between $N_{-}(a)$ and the previously identified curve $N_{*}(a)$ Eq. (36), which now applies for this smaller range of $a$ (the dotted curve bounding regions II and III). Furthermore, out-of-plane steady states occur precisely on these two boundaries. The essential difference between the upper and lower nematic equilibria in weak shear is that existence for the lower nematic branch occurs for all aspect ratios, consistent with results in [Forest and Wang (2002)].

\section{RHEOLOGICAL PROPERTIES OF STABLE STEADY STATES}

The stable, robust steady states that have been characterized in detail by our analysis consist of the nearly isotropic, flow-aligned solutions in the dilute concentration regime, labeled FA0, Fig. 1(b), and the in-plane, flow-aligned nematic solutions in the intermediate concentration regime, labeled FA1, Fig. 1(b). (The out-of-plane flow-aligned states are rare, and not likely to be experimentally observed.) Rheological properties of these attractors follow directly from the explicit formulas for $\mathbf{Q}=\mathbf{Q}_{0}+\mathrm{Pe} \mathbf{Q}_{1}$, given earlier. For the Doi theory, the stress tensor is given by Wang (2002):

$$
\begin{aligned}
\tau= & {\left[\frac{2}{\operatorname{Re}}+3 \nu k T \zeta_{3}(a)\right] \mathbf{D}+3 a \nu k T\left[\mathbf{Q}-N\left(\mathbf{Q}+\frac{\mathbf{I}}{3}\right) \mathbf{Q}+N \mathbf{Q}:\langle\mathbf{m m m m}\rangle\right] } \\
& +3 \nu k T\left[\zeta_{1}(a)(\mathbf{D M}+\mathbf{M D})+\zeta_{2}(a) \mathbf{D}:\langle\mathbf{m m m m}\rangle\right],
\end{aligned}
$$

where

$$
\begin{gathered}
\zeta_{3}=\frac{\zeta^{(0)}}{I_{1}}, \quad \zeta_{1}=\zeta^{(0)}\left(\frac{1}{I_{3}}-\frac{1}{I_{1}}\right), \quad \zeta_{2}=\zeta^{(0)}\left[\frac{J_{1}}{I_{1} J_{3}}+\frac{1}{I_{1}}-\frac{2}{I_{3}}\right], \\
r=\sqrt{\frac{1+a}{1-a}}, \quad I_{1}=2 r \int_{0}^{\infty} \frac{d x}{\sqrt{\left(r^{2}+x\right)}(1+x)^{3}}, \\
I_{3}=r\left(r^{2}+1\right) \int_{0}^{\infty} \frac{d x}{\sqrt{\left(r^{2}+x\right)}(1+x)^{2}\left(r^{2}+x\right)}, \\
J_{1}=r \int_{0}^{\infty} \frac{x d x}{\sqrt{\left(r^{2}+x\right)}(1+x)^{3}}, \quad J_{3}=r \int_{0}^{\infty} \frac{x d x}{\sqrt{\left(r^{2}+x\right)}(1+x)^{2}\left(r^{2}+x\right)},
\end{gathered}
$$

$\zeta^{(0)}$ is a material parameter which must be experimentally measured, and $\nu$ is the number density of LCP molecules per unit volume. The apparent viscosity $\eta$, first and second normal stress differences $N_{1}$ and $N_{2}$, are given by

$$
\begin{gathered}
N_{1}=\tau_{x x}-\tau_{y y}, \\
N_{2}=\tau_{y y}-\tau_{z z}, \\
\eta=\tau_{x y} / \mathrm{Pe} .
\end{gathered}
$$

After scaling by $3 \nu k T \zeta^{(0)}, N_{1,2}$ are depicted in Fig. 6, Fig. 7 for fixed aspect ratio $a$ $=0.8(r=3)$ and shear rate $\mathrm{Pe}=0.1$, then in Fig. 8 for variable aspect ratio. For $\eta$, the viscous contribution is assumed negligible and we use $\zeta^{(0)}=0.04$. 

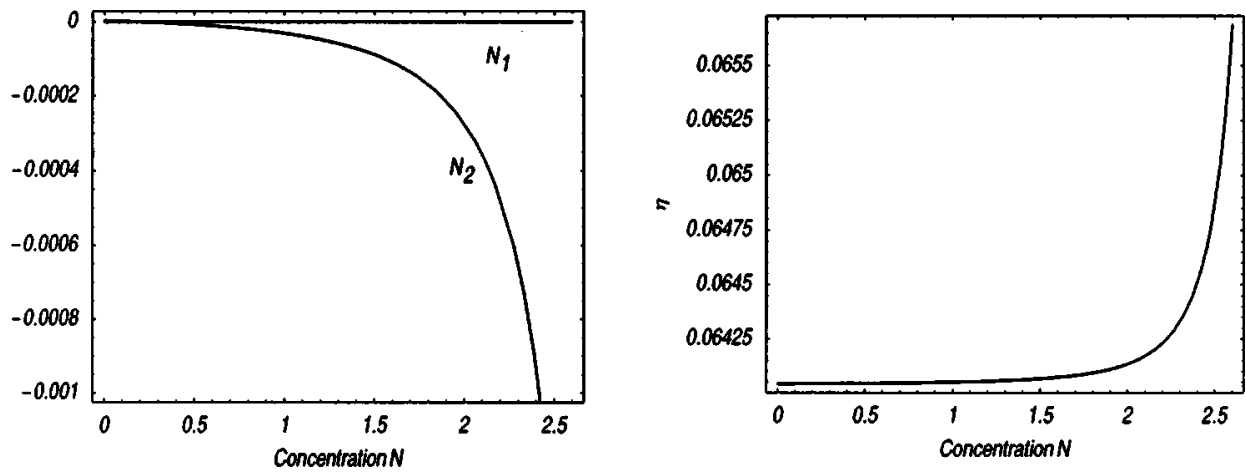

FIG. 6. Dilute concentration regime. First and second normal stress differences $N_{1}$ and $N_{2}$, and the apparent viscosity $\eta$ vs nematic concentration $N$ for the stable, nearly isotropic, flow aligned states FA0 of Fig. 1(b). The parameters chosen are $a=0.8$, or aspect ratio $r=3$, and normalized shear rate $\mathrm{Pe}=0.1$.

In the dilute concentration regime, where the nearly isotropic, flow-aligned state is the unique attractor: $N_{1}=0 ; N_{2}$ is small but increasingly negative as $N$ increases; the apparent viscosity is constant for very low $N$ then increases as the instability transition is approached. We are not aware of experimental data to benchmark this prediction. These features are illustrated in Fig. 6.

At all nematic concentrations with stable flow-aligned equilibria, region I of Fig. 1 and Table I:

- $N_{1}>0$ and $N_{2}<0$, which is consistent with experimental data for flow-aligned nematic polymers in weak shear [cf., Moldenaers and Mewis (1993), Burghardt (1998), Larson (1998)].

- The scaling behavior of the first and second normal stress differences versus nematic concentration $N$, for a fixed aspect ratio and shear rate, is conveyed by Fig. 7(a). The dots correspond to numerical solution data of the Doi model, showing excellent agreement with our theory except for an $O(\mathrm{Pe})$ neighborhood of $N=\frac{8}{3}$. Both $N_{1}$ and $N_{2}$ are monotone versus $N$ and approach 0 at the critical concentration $N_{*}(a)$, Eq. (36),
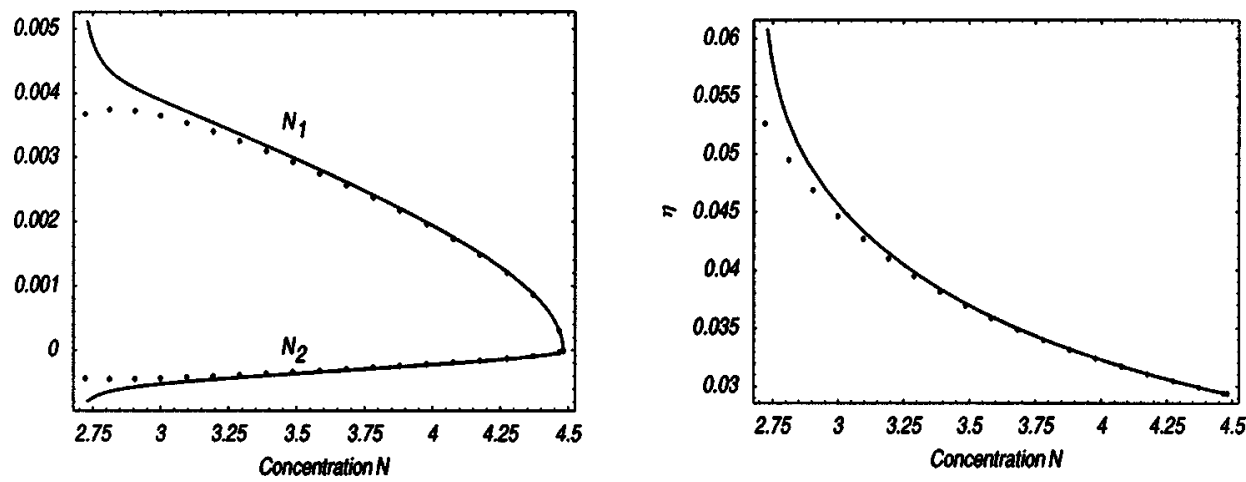

FIG. 7. Nematic concentration regime. First and second normal stress differences and apparent viscosity for the stable, in-plane, shear-aligned state FA1, Fig. 1(b), selected from the stable quiescent nematic solution, for all nematic concentrations $N>\frac{8}{3}$. The molecular aspect ratio is $r=3$, and the normalized shear rate is Pe $=0.1$. 

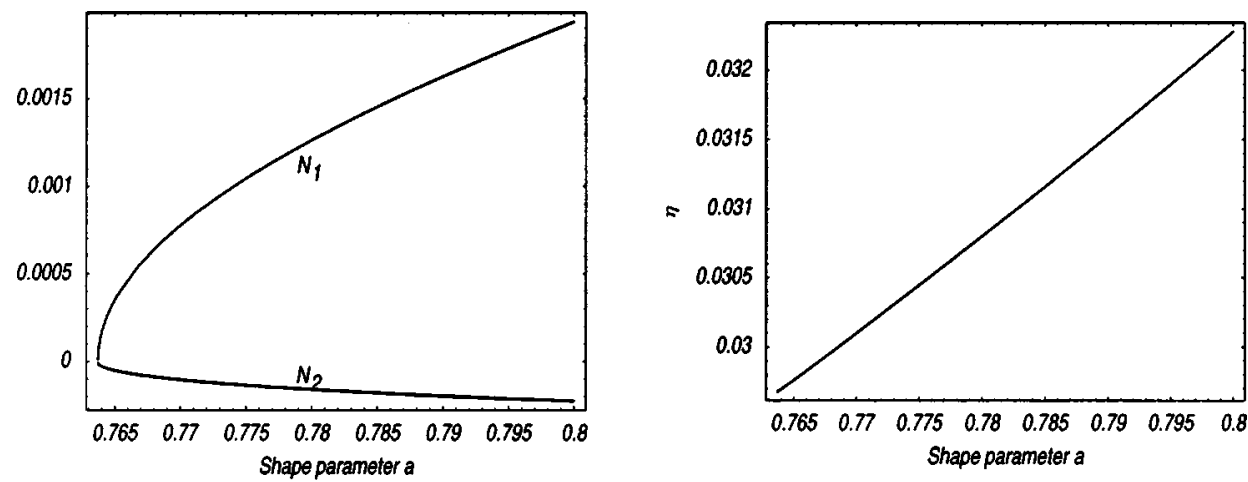

FIG. 8. Variations in rheological properties due to molecular aspect ratio. Normal stress differences $\left(N_{1}, N_{2}\right)$ and apparent viscosity $(\eta)$ vs shape parameter $a$ for the stable flow aligned states FA1 of Fig. 1(b). The parameters chosen are $N=4$, and normalized shear rate $\mathrm{Pe}=0.1$.

for the onset of unsteady response! This result suggests a sign change in $N_{1}$ and $N_{2}$, or even oscillations in their sign, as the unsteady (kayaking) state emerges at slightly higher concentrations.

- The apparent viscosity Fig. 7(b) exhibits viscous thinning over this range of concentrations, analogous to the shear-rate data from [Burghardt (1998)], Figs. 7 and 8. The dots are numerical solution data. The orientational viscosity is low as the steadyunsteady transition is approached; this prediction is natural given the tendency illustrated in Fig. 2 for the Leslie alignment angle to approach the flow axis as $N$ approaches the unsteady transition.

- The standard rheological data on sign changes in $N_{1}, N_{2}$ correspond to shear-rate transitions. This result implies sign changes in normal stress differences are also associated with concentration-induced, steady-unsteady transitions at very low shear rates.

- Scaling behavior of normal stresses and apparent viscosity with respect to molecular aspect ratio are conveyed in Fig. 8. Note that if one lowers the aspect ratio to the lower limit where flow-alignment states are lost, once again both normal stress differences approach zero, indicating a sign change. Generally, all three rheological features increase with aspect ratio, with only $N_{1}$ indicating a measurable difference.

\section{NUMERICAL CONFIRMATION}

Alignment and rheology of several stable steady solutions have been numerically confirmed in the discussions and figures earlier. We now address the analytical predictions of our method regarding more global information: phase transitions and bifurcation curves versus mesoscopic material parameters $N$ and $r$. Remarkably, our solvability conditions for three separate solutions (stability of logrolling states, persistence of inplane flow-alignment, and existence of out-of-plane steady states) have all identified the same Doi closure model criterion: $\left|\lambda_{L}\right|=|[a(2+s)] / 3 s|=1$. When applied to the quiescent stable nematic $s_{+}(N)$ and to the unstable quiescent nematic $s_{-}(N)$, we get the transition curves in Fig. 1.

We now numerically confirm the phase transition results in Fig. 1, for two disparate aspect ratios, by computing the bifurcation diagrams versus nematic concentration $N$ as in [Faraoni et al. (1999), Forest and Wang (2002)]. First, we fix $r=3$, or $a=\frac{4}{5}$ $>\frac{1}{3}$, and compute all steady solutions of the full Doi closure model versus $N$, at fixed weak shear rate $\mathrm{De}=0.01$, where $\mathrm{De}=6 \mathrm{Pe}$. We then choose a less extreme, nearly 

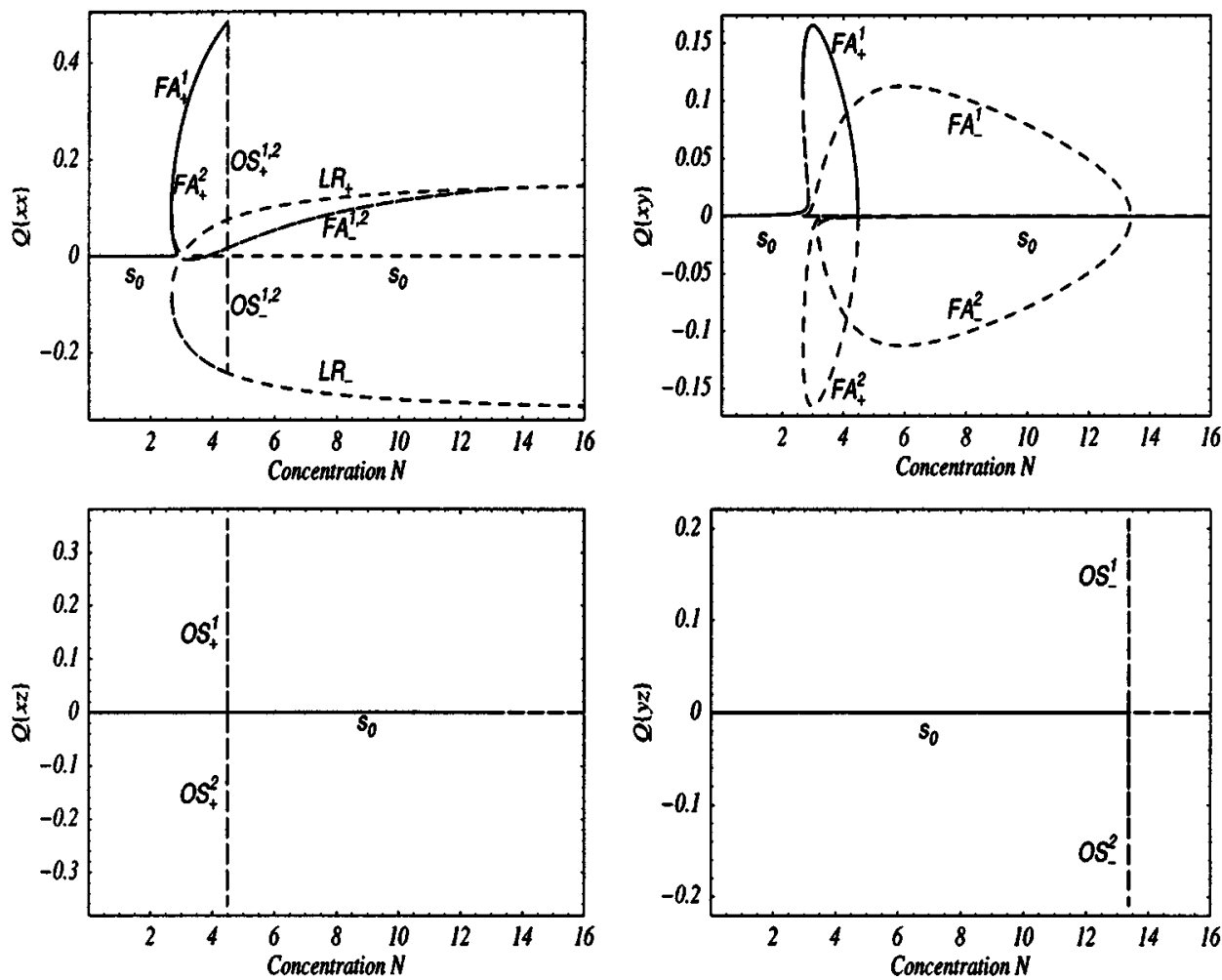

FIG. 9. Numerical bifurcation diagram of steady solutions for the Doi closure model with aspect ratio $r$ $=3(a=.8)$, and weak normalized shear rate $\mathrm{De}=0.01, \mathrm{De}=6 \mathrm{Pe}$. Solutions are depicted vs nematic concentration $N$. Out-of-plane solutions emerge at critical concentrations $N$ within $O\left(\mathrm{Pe}^{2}\right)$ of theoretical predictions, $N=\frac{121}{27}$ and $N=\frac{361}{27}$. In-plane states are distinguished by zero out-of-plane components, $Q(x z)$ $=Q(y z)=0$. The solid line represents the only stable branch of solutions.

spherical, aspect ratio $r=\sqrt{\frac{3}{2}}$, or $a=\frac{1}{5}<\frac{1}{3}$. The bifurcation diagrams projected onto four tensor components are shown in Figs. 9 and 10. For both aspect ratios, the termination of the in-plane solution and the emergence of the out-of-plane solution agree with theory to within $O\left(\mathrm{Pe}^{2}\right)$.

\section{CONCLUSION}

Weak-shear steady state criteria have been derived for general mesoscopic tensor models, giving the mesoscopic analog of the Leslie tumbling criterion of continuum theory, and of the Kuzuu-Doi weak-flow analysis of kinetic theory. These full-tensor conditions characterize in detail all steady solutions which survive in the weak shear limit from the quiescent equilibrium set, and their explicit dependence on mesoscopic theory parameters. We choose the Doi quadratic closure example to work out the full consequences of our method; the number, type, stability, and phase transitions of steady orientational distributions versus nematic concentration $N$ and molecular aspect ratio $r$ are derived with explicit formulas. The number of steady states varies between 3 and 7; the subset of stable steady states is between 0 and 2, either in-plane flow-aligned or out-ofplane aligned states, and possibly a stable logrolling state only at extremely high concentrations $N \gg 1$. The method can be applied to any mesoscopic tensor model; the requisite 

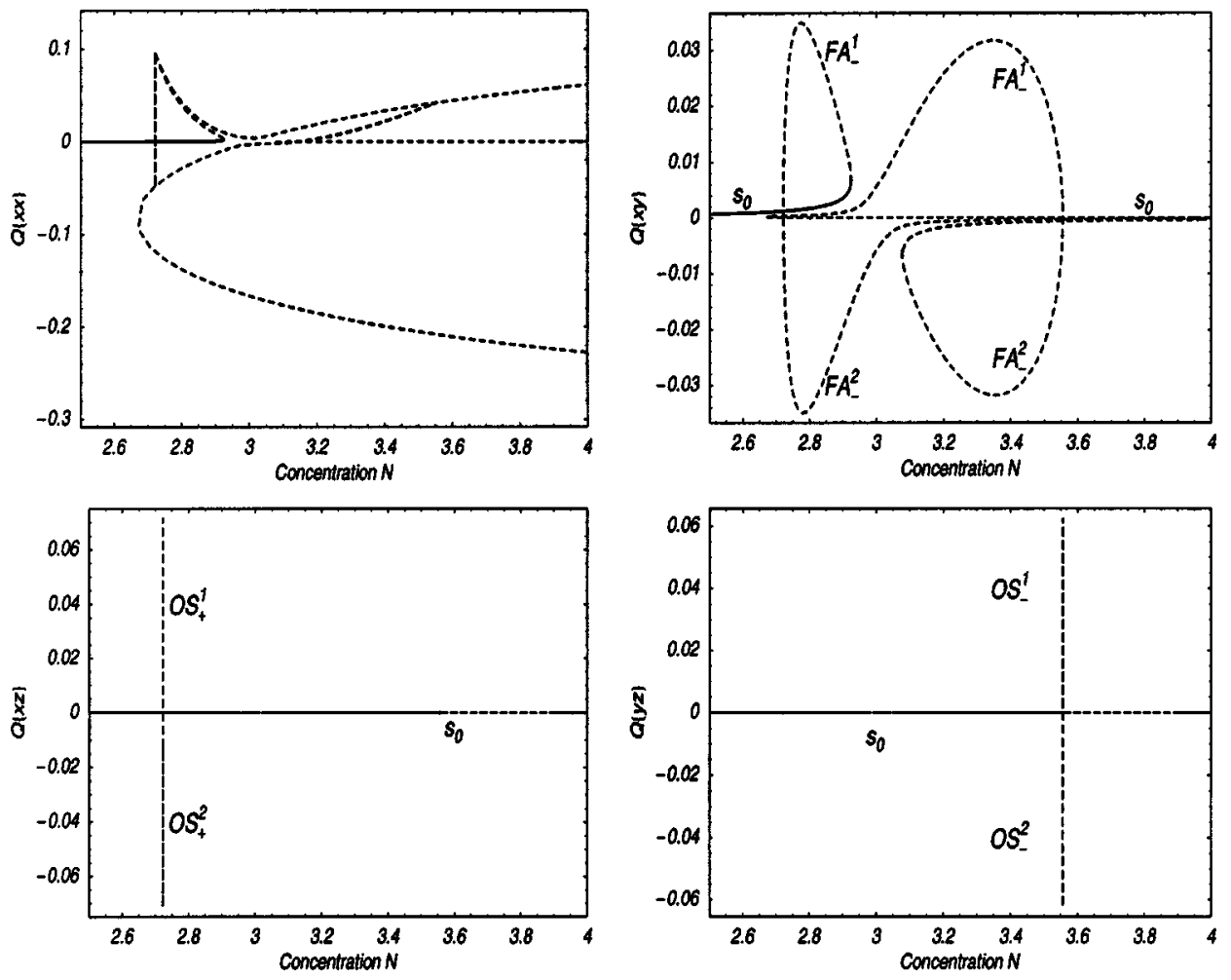

FIG. 10. Numerical bifurcation diagram of the Doi closure model for nearly spherical aspect ratio $r=\sqrt{\frac{3}{2}}$ $(a=0.2)$, and weak normalized shear rate $\mathrm{De}=0.01$. The out-of-plane solutions emerge at $N=2.722229$ and 3.555548 , whereas the theory predicts $N=\frac{49}{38}$ and $N=\frac{32}{9}$, which agree to well within $O\left(\mathrm{Pe}^{2}\right)$.

analysis consists of solving five-dimensional linear systems in closed form, which we outline for the Doi closure model in the Appendix. Our analysis is confirmed, qualitatively and quantitatively, with numerical flow-phase bifurcation diagrams. Furthermore, our explicit construction of all robust stable alignment states yields all measurable laboratory properties (alignment angle, birefringence, first and second normal stress differences, apparent viscosity), and their scaling behavior with respect to nematic concentration and molecular aspect ratio. These predictions allow one to extrapolate from laboratory measurements of shear-rate scaling properties. We have matched the Doi model predictions in qualitative terms with experimental data summarized by Burghardt (1998).

See Supplementary Material in EPAPS Document No. E-JORHD2-47-009301. Information on accessing this document is contained at the beginning of the reference section.

\section{APPENDIX A: SOLVABILITY CONDITION FOR $Q_{2}$}

The tensor equation for $\mathbf{Q}_{2}$ is

$$
\overline{\mathbf{A}}\left(\mathbf{Q}_{0}, \mathbf{Q}_{1}\right) \cdot \mathbf{Q}_{2}=\overline{\mathbf{r}}\left(\mathbf{Q}_{0}, \mathbf{Q}_{1}\right),
$$

where 


$$
\begin{aligned}
& \overline{\mathbf{A}}\left(\mathbf{Q}_{0}, \mathbf{Q}_{1}\right) \cdot \mathbf{Q}_{2}=\mathbf{Q}_{2}-N\left(\mathbf{M}_{0} \cdot \mathbf{Q}_{2}+\mathbf{Q}_{2} \cdot \mathbf{Q}_{0}\right)+N\left[\mathbf{Q}_{0}: \mathbf{M}_{0} \mathbf{Q}_{2}+\left(\mathbf{Q}_{2}: \mathbf{M}_{0}+\mathbf{Q}_{0}: \mathbf{Q}_{2}\right) \mathbf{M}_{0}\right] \\
& \overline{\mathbf{r}}\left(\mathbf{Q}_{0}, \mathbf{Q}_{1}\right)= \tilde{\mathbf{\Omega}} \cdot \mathbf{Q}_{1}-\mathbf{Q}_{1} \cdot \tilde{\mathbf{\Omega}}+a\left(\tilde{\mathbf{D}} \cdot \mathbf{Q}_{1}+\mathbf{Q}_{1} \cdot \tilde{\mathbf{D}}\right)-2 a\left(\tilde{\mathbf{D}}: \mathbf{M}_{0} \mathbf{Q}_{1}+\tilde{\mathbf{D}}: \mathbf{M}_{1} \mathbf{M}_{0}\right)+N \mathbf{Q}_{1} \cdot \mathbf{Q}_{1} \\
&-N\left[\left(\mathbf{Q}_{1}: \mathbf{M}_{0}+\mathbf{Q}_{0}: \mathbf{Q}_{1}\right) \mathbf{Q}_{1}+\mathbf{Q}_{1}: \mathbf{Q}_{1} \mathbf{M}_{0}\right] .
\end{aligned}
$$

The solvability condition for $\mathbf{Q}_{2}$ therefore is

$$
\mathbf{r}\left(\mathbf{Q}_{0}, \mathbf{Q}_{1}\right) \perp \mathcal{N}\left[\overline{\mathbf{A}}^{\operatorname{adj}}\left(\mathbf{Q}_{0}, \mathbf{Q}_{1}\right)\right] .
$$

\section{APPENDIX B: ANALYSIS OF THE STEADY SOLVABILITY CONDITIONS}

See Supplementary Material in EPAPS Document No. E-JORHD2-47-009301.

\section{References}

See EPAPS Document No. E-JORHD2-47-009301 for a discussion of full-tensor criteria for sheared nematic polymers. A direct link to this document may be found in the article's HTML reference section. The document may also be retrieved via the EPAPS home page (http://www.aip.org.pubservs.epaps.html) or from ftp.aip.org in the directory /epaps/. See the EPAPS homepage for more information.

Beris, A. N., and B. J. Edwards, Thermodynamics of Flowing Systems with Internal Microstructure (Oxford Science, New York, 1994).

Burghardt, W. R., "Molecular orientation and rheology in sheared lyotropic liquid crystalline polymers," Macromol. Chem. Phys. 199, 471-488 (1998).

Chaubal, C. V., L. G. Leal, and G. H. Fredrickson, "A comparison of closure approximations for the Doi theory of LCPs," J. Rheol. 39, 73-103 (1995).

Chillingworth, D. R. J., E. Vicente Alonso, and A. A. Wheeler, "Geometry and dynamics of a nematic liquid crystal in a uniform shear flow," J. Phys. A 34, 1393-1404 (2001).

Cocchini, F., C. Aratari, and G. Marrucci, "Tumbling of rodlike polymers in the liquid-crystalline phase under shear flow," Macromolecules 23, 4446-4451 (1990).

de Gennes, P.-G., and J. Prost, The Physics of Liquid Crystals (Oxford University Press, New York, 1993).

Doi, M., "Molecular dynamics and rheological properties of concentrated solutions of rodlike polymers in isotropic and liquid crystalline phases," J. Polym. Sci., Polym. Phys. Ed. 19, 229-243 (1981).

Doi, M., and S. F. Edwards, The Theory of Polymer Dynamics (Oxford University Press, London, 1986).

Ericksen, J. L., “Anisotropic fluids,” Arch. Ration. Mech. Anal. 4, 231-237 (1960).

Faraoni, V., M. Grosso, S. Crescitelli, and P. L. Maffettone, "The rigid-rod model for nematic polymers: An analysis of the shear flow problem," J. Rheol. 43, 829-843 (1999).

Feng, J., G. Sgalari, and G. Leal, "A theory for flowing nematic polymers with orientational distortions," J. Rheol. 44, 1085-1101 (2000).

Forest, M. G., and Q. Wang, "Monodomain response of finite-aspect-ratio macromolecules in shear and related linear flows," Rheol. Acta (in press).

Forest, M. G., Q. Wang, and H. Zhou, "Homogeneous pattern selection and director instabilities of nematic liquid crystal polymers induced by elongational flows," Phys. Fluids 12, 490-498 (2000).

Forest, M. G., Q. Wang, and H. Zhou, "Structure evolution in tumbling and kayaking nematic LCPs between shearing plates," UNC Program in Applied Mathematics Preprint No. PAMPS2002-2 (2002a), available from http://www.amath.unc.edu/PAMPS/

Forest, M. G., Q. Wang, and R. Zhou, "Symmetries of the Doi kinetic theory for nematic polymers of arbitrary aspect ratio: at rest and in linear flows," Phys. Rev. E 66, 031712 (2002b).

Forest, M. G., Q. Wang, and R. Zhou, "The weak shear phase diagram for nematic polymers," UNC Program in Applied Mathematics Preprint No. PAMPS2002-16 (2002c), available from http://www.amath.unc.edu/ PAMPS/.

Forest, M. G., Q. Wang, and R. Zhou, "Explicit flow-aligned orientational distribution functions for dilute nematic polymers in weak shear," UNC Program in Applied Mathematics Preprint No. PAMPS2002-26 (2002d), available from http://www.amath.unc.edu/PAMPS/.

Fuller, G. G., Optical Rheometry of Complex Fluids (Oxford University Press, Oxford, 1995).

Grecov, D., and A. Rey, "Theoretical and computational rheology for discotic nematic liquid crystals," Mol. Cryst. Liq. Cryst. (in press). 
Hess, S., "Fokker-Planck-equation approach to flow alignment in liquid crystals," Z. Naturforsch. Teil A 31A, 1034 (1976).

Hinch, E. J., and L. G. Leal, “Constitutive equations in suspension mechanics, part 2, Approximate forms for a suspension of rigid particles affected by Browian rotations," J. Fluid Mech. 76, 187-208 (1976).

Kuzuu, N., and M. J. Doi, "Constitutive equation for nematic liquid crystals under weak velocity gradient derived from a molecular kinetic equation,” J. Phys. Soc. Jpn. 52, 3486-3494 (1983).

Kuzuu, N., and M. J. Doi, "Constitutive equation for nematic liquid crystals under weak velocity gradient derived from a molecular kinetic equation, II. Leslie coefficients for rodlike polymers,” J. Phys. Soc. Jpn. 53, 1031-1040 (1984).

Landau, L. D., in Collected Papers, edited by S. Kranse (Gordon and Breach, New York, 1965), p. 193.

Larson, R. G., "Arrested tumbling in shearing flows of liquid crystal polymers," Macromolecules 23, 39833992 (1990).

Larson, R. G., Rheology of Complex Fluids (Oxford University Press, New York, 1998).

Larson, R. G., and H. Ottinger, "The effect of molecular elasticity on out-of-plane orientations in shearing flows of liquid crystalline polymers," Macromolecules 24, 6270-6282 (1991).

Leslie, F. M., "Some constitutive equations for liquid crystals," Arch. Ration. Mech. Anal. 28, 265-283 (1968).

Maffettone, P. L., A. M. Sonnet, and E. G. Virga, "Shear-induced biaxiality in nematic polymers," J. NonNewtonian Fluid Mech. 90, 283-297 (2000).

Marrucci, G., and N. Grizzuti, Paper presented at the 59th Annual Meeting of the Society of Rheology, Atlanta, GA, October 1987.

Marrucci, G., "Tumbling regime of liquid crystalline polymers," Macromolecules 24, 4176-4182 (1991).

Marrucci, G., and F. Greco, "The elastic constants of Maier-Saupe rodlike molecular nematics," Mol. Cryst. Liq. Cryst. 206, 17-30 (1991).

Marrucci, G., and F. Greco, "Flow behavior of liquid crystalline polymers," Adv. Chem. Phys. 86, 331-404 (1993).

Marrucci, G., and P. L. Maffettone, "Description for the liquid crystalline phase of rodlike polymers at high shear rates," Macromolecules 22, 4076-4082 (1989).

Mather, P. T., D. S. Pearson, and R. G. Larson, "Flow patterns and disclination-density measurements in sheared nematic liquid crystals, I. Flow-aligning 5CB," Liq. Cryst. 20, 527-538 (1996a).

Mather, P. T., D. S. Pearson, and R. G. Larson, "Flow patterns and disclination-density measurements in sheared nematic liquid crystals, II. Tumbling 8CB," Liq. Cryst. 20, 539-546 (1996b).

Mewis, J., M. Mortier, J. Vermant, and P. Moldenaers, "Experimental evidence for the existence of a wagging regime in polymeric liquid crystals," Macromolecules 30, 1323-1328 (1997).

Moldenaers, P., and J. Mewis, "On the nature of viscoelasticity in polymeric liquid crystals," J. Rheol. 37, 367-380 (1993).

Rienacker, G., and S. Hess, "Orientational dynamics of nematic liquid crystals under shear flow," Physica A 267, 294-321 (1999).

Tsuji, T., and A. D. Rey, "Effect of long range order on sheared liquid crystalline polymers, Part 1: Compatibility between tumbling behavior and fixed anchoring," J. Non-Newtonian Fluid Mech. 73, 127-152 (1997).

Ugaz, V. M., R. Burghardt, W. Zhou, and J. A. Kornfield, "Transient molecular orientation and rheology in flow aligning thermotropic liquid crystalline polymers," J. Rheol. 45, 1029-1062 (2001).

Van Horn, B. L., and H. H. Winter, "Dynamics of shear aligning of nematic liquid crystal monodomains," Rheol. Acta 39, 294-300 (2000).

Wang, Q., "Biaxial steady states and their stability in shear flows of liquid crystal polymers," J. Rheol. 41, 943-970 (1997).

Wang, Q., “A hydrodynamic theory for solutions of nonhomogeneous nematic liquid crystalline polymers of different configuration," J. Chem. Phys. 116, 9120-9136 (2002).

Zhou, W. J., J. Kornfield, and W. Burghardt, "Shear alignment properties of a main chain thermotropic liquid crystalline polymer," Macromolecules 34, 3654-3660 (2001). 